\title{
Prognostic factors influencing survival in 35 patients with malignant peritoneal mesothelioma
}

\author{
H. KAYA ${ }^{1, \star}$, C. SEZGI ${ }^{1}$, A. C. TANRIKULU ${ }^{1}$, M. TAYLAN ${ }^{1}$, O. ABAKAY ${ }^{1}$, H. S. SEN ${ }^{1}$, A. ABAKAY ${ }^{1}$, M. KUCUKONER ${ }^{2}$, M. KAPAN ${ }^{3}$ \\ ${ }^{1}$ Department of Chest Diseases, Faculty of Medicine, Dicle University, Diyarbakir, Turkey; ${ }^{2}$ Department of Medical Oncology, Faculty of Medicine, \\ Dicle University, Diyarbakir, Turkey; ${ }^{3}$ Department of General Surgery, Faculty of Medicine, Dicle University, Diyarbakir, Turkey
}

*Correspondence: halidekaya@yahoo.com.tr

Received August 28, 2013 / Accepted November 14, 2013

\begin{abstract}
Malignant mesothelioma is a rare but highly lethal form of cancer that affects the serosal membranes. Malignant peritoneal mesothelioma (MPM) is the second most common form of malignant mesothelioma (pleural mesothelioma is the most common). The aim of this study was to evaluate prognostic factors influencing the survival of patients with MPM.

A retrospective analysis was performed on 35 patients who were admitted to our hospital between March 2005 and July 2013. The patients' demographic and clinical data, laboratory results, radiological signs, Eastern Cooperative Oncology Group (ECOG) performance status (PS), and treatment outcomes were evaluated.

The mean age of the 35 patients was $59.0 \pm 14$.4 years, the mean survival time was $16.2 \pm 12.9$ months, and the majority of the histopathological types of MPM were epithelial (68.6\%). $82.9 \%$ of the patients had been exposed to asbestos, and the mean duration of exposure was $28.3 \pm 14.5$ years. The most frequent symptoms were abdominal distention/pain, weight loss, dyspnea, and chest pain. The mean interval between the onset of symptoms and the diagnosis was $4.6 \pm 3.3$ months. Platinum-based combination chemotherapy in combination with supportive care was used in the treatment of $68.6 \%$ of the patients, while supportive treatment alone was used in the others. Our results revealed that patients who were $>60$ years old ( $p=0.019)$, who were exposed to asbestos $>20$ years $(p=0.033)$, who had an ECOG PS of $3(p=0.000)$ were more likely to have a poor MPM prognosis.

In conclusion, increased age, duration of environmental asbestos exposure and ECOG PS are important factors that influence the prognosis of MPM patients.
\end{abstract}

Key words: Malignant peritoneal mesothelioma, prognostic factors, survival

Malignant mesothelioma is a rare but aggressive and fatal type of cancer that develops from the mesothelial cells of serosal membranes (pleura, peritoneum, pericardium or tunica vaginalis testes). Malignant peritoneal mesothelioma (MPM) occurs in the second order among all mesotheliomas. It accounts for about 10 to $15 \%$ of all cases diagnosed with mesothelioma in the United States [1]. The inhalation of asbestos fibers is the most frequent and significant etiologic factor in all forms of mesothelioma. Although a strong relationship between asbestos exposure and development of pleural mes-

\footnotetext{
Abbreviations: ALP: Alkaline phosphatase, CRP: C-reactive protein, CT: Computed tomography, ECOG: Eastern Cooperative Oncology Group, ESR: Erythrocyte sedimentation rate, $\mathrm{Hb}$ : Hemoglobin concentration, $\mathrm{LDH}$ : Lactate dehydrogenase, MPM: Malignant peritoneal mesothelioma, PS: Performance status, US: Ultrasound, WBC: White blood cell count
}

othelioma has been shown, such a strong relationship has not been found with MPM [2]. In addition, although they share asbestos exposure as a common risk factor, MPM and pleural mesothelioma are very different [3]. Most of the published literature examining the prognostic factors of mesothelioma are regarding pleural mesothelioma, but knowledge about MPM is limited [4]. Most patients with MPM have a poor prognosis and survival time ranges between 7 and 13.5 months. Since primary malignancies developing from the peritoneum are very rare, there is no widely and easily applicable prognostic scoring system for MPM (although there is one for pleural mesothelioma) [5].

In the present study, demographic and clinical data, laboratory results, radiological signs, and features of systemic chemotherapy were evaluated in 35 patients who were diagnosed with MPM and questioned regarding their histories of 
environmental asbestos exposure. We also determined which prognostic factors impacted survival.

\section{Patients and methods}

A retrospective analysis was performed on 35 patients who were admitted to our hospital between March 2005 and July 2013. The patients' demographic and clinical data, laboratory results, radiological signs, Eastern Cooperative Oncology Group (ECOG) performance status (PS), and treatment outcomes were defined as potential prognostic factors and were measured at the time of diagnosis. A histopathological evaluation was performed on all surgical (laparotomy/laparoscopy) or percutaneous peritoneal needle biopsies and/or on any paracentesis materials.

Clinical features. The following pretreatment characteristics were analyzed for their impact on survival: age at the time of diagnosis ( $\leq 60$ or $>60$ years), gender, environmental asbestos exposure (yes or no), duration of exposure ( $\leq 20$

Table 1. Demographic, clinical, and laboratory features of the malignant peritoneal mesothelioma patients

\begin{tabular}{|c|c|}
\hline Age (mean \pm sd, years) & $59.0 \pm 14.4$ \\
\hline Age group ( $\leq 60 />60$ years $)$ & $22 / 13$ \\
\hline Gender (female/male) & $19 / 16$ \\
\hline Asbestos exposure (n,\%) & $29(82.9)$ \\
\hline Duration of asbestos exposure (mean \pm sd, years) & $28.3 \pm 14.5$ \\
\hline History of smoking $(\mathrm{n}, \%)$ & $13(37.1)$ \\
\hline \multicolumn{2}{|l|}{ Symptoms at diagnosis (n,\%) } \\
\hline Abdominal distention/pain & $34(97.1)$ \\
\hline Weight loss & $17(48.6)$ \\
\hline Dyspnea & $15(42.9)$ \\
\hline Chest pain & $8(22.9)$ \\
\hline \multicolumn{2}{|l|}{ Laboratory results (mean \pm sd) } \\
\hline Hemoglobin concentration (g/dl) & $11.4 \pm 1.8$ \\
\hline White blood cell count $\left(\times 10^{3} / \mu \mathrm{l}\right)$ & $9.8 \pm 4.0$ \\
\hline Platelet count $\left(\mathrm{x} 10^{3} / \mu \mathrm{l}\right)$ & $404.7 \pm 160.4$ \\
\hline Serum albumin level (g/dl) & $2.7 \pm 0.7$ \\
\hline Lactate dehydrogenase (U/l) & $325.4 \pm 317.4$ \\
\hline Alkaline phosphatase (U/l) & $128.8 \pm 95.6$ \\
\hline C-reactive protein $(\mathrm{mg} / \mathrm{l})$ & $58.8 \pm 39.6$ \\
\hline Erythrocyte sedimentation rate $(\mathrm{mm} / \mathrm{h})$ & $59.8 \pm 25.5$ \\
\hline \multicolumn{2}{|l|}{ Histopathological type (n,\%) } \\
\hline Epithelial & $24(68.6)$ \\
\hline Others & $11(31.4)$ \\
\hline \multicolumn{2}{|l|}{ Performance status (ECOG) $(n, \%)$} \\
\hline 1 & $17(48.6)$ \\
\hline 2 & $12(34.3)$ \\
\hline 3 & $6(17.1)$ \\
\hline Systemic chemotherapy (n,\%) & $24(68.6)$ \\
\hline \multicolumn{2}{|l|}{ Status (n, \%) } \\
\hline Alive & $3(8.6)$ \\
\hline Dead & $32(91.4)$ \\
\hline
\end{tabular}

ECOG: Eastern Cooperative Oncology Group. or $>20$ years), history of smoking (yes or no), pack-years of smoking $(\leq 20$ or $>20)$, symptoms at the time of diagnosis [presence of abdominal distention/pain, weight loss ( $>5 \%$ in the last 3 months), chest pain, dyspnea], the interval between the onset of symptoms until the diagnosis ( $\leq 6$ or $>6$ months), diagnostic procedures, and histopathological types [epithelioid or others (biphasic, sarcomatous and unspecified)]. Performance status was assessed using an ECOG score from 0 to 5 , with 0 indicating asymptomatic, 1 indicating symptomatic but completely ambulatory, 2 indicating symptomatic with $<50 \%$ of time spent in bed during the day, 3 indicating symptomatic with $>50 \%$ of time spent in bed but not bedridden, 4 indicating bedridden and 5 indicating death [6]. The patients who received systemic chemotherapy were further split into two groups, with the first group receiving pemetrexed and cisplatin and the second group receiving gemcitabine and cisplatin. The interval between the diagnosis and death or the last visit (months) was recorded.

Laboratory results. The following laboratory parameters were determined and recorded: hemoglobin concentration $(\mathrm{Hb},<12.30$ or $\geq 12.30 \mathrm{~g} / \mathrm{dl}$ ), white blood cell count (WBC, $\leq 11,300$ or $>11,300 / \mu \mathrm{l})$, platelet count $(\leq 420,000$ or $>420,000 /$ $\mu \mathrm{l})$, levels of serum albumin $(\leq 3.0 \mathrm{or}>3.0 \mathrm{~g} / \mathrm{dl})$, lactate dehydrogenase (LDH, $\leq 500$ or $>500 \mathrm{U} / \mathrm{l})$, alkaline phosphatase (ALP, $\leq 79$ or $>79 \mathrm{U} / 1$ ), C-reactive protein (CRP, $\leq 50$ or $>50 \mathrm{mg} / \mathrm{l}$ ), and erythrocyte sedimentation rate (ESR, $\leq 50$ or $>50 \mathrm{~mm} / \mathrm{h}$ ).

Radiological signs. All radiological imaging recorded in our database, including abdominal and/or transthoracic ultrasound (US) and computed tomography (CT) was evaluated. Initial CT results (abdominal and thoracic changes) of MPM patients were classified as follows: the presence of ascites, peritoneal, omental and mesenteric involvement, abdominal organ involvement (liver, spleen), abdominal adenopathy, pleural fluid, pleural calcification/plaques and pleural thickening.

Statistical analysis. The data collected were analyzed using SPSS for Windows version 15.0. The patient characteristics were reported using frequency and descriptive analysis. Survival analysis was performed using the Kaplan-Meier method and confounding factors related to survival were analysed using the stratified Log Rank test. Duration of survival was defined as the period between diagnosis and death, and for patients who did not die, this duration was defined as the period between the time of diagnosis and July 2013. P values $<0.05$ were considered statistically significant.

\section{Results}

Patient characteristics. Baseline characteristics for the 35 patients are summarized in Table 1 . Their mean age was $59.0 \pm 14.4$ years (range $36-92$ ), and 13 were older than 60 years. The mean duration of asbestos exposure was $28.3 \pm 14.5$ years (range 5 - 70). The most frequent initial symptom $(97 \%$ of the patients) was abdominal distention/pain. The mean interval between the onset of symptoms and the diagnosis 
was 4.6 \pm 3.3 months (range 1-12). Seventeen (48.6\%) patients underwent surgical diagnostic procedures, 18 (51.4\%) underwent percutaneous peritoneal needle biopsy, and 21 patients (60\%) were diagnosed by paracentesis materials. ECOG PS of the patients scored 1, 2 or 3 at the time of diagnosis (48.6\%, $34.3 \%, 17.1 \%$, respectively). A combination of platinum-based combination chemotherapy and supportive care was used in the treatment of $68.6 \%$ of the patients.

Laboratory results. There was no significant prognostic relationship between survival and the pretreatment levels of complete blood count ( $\mathrm{Hb}, \mathrm{WBC}$ and platelets), serum albumin, LDH, ALP, CRP, and ESR ( $>$ >0.05).

Radiological signs. The radiological signs of the 35 patients are summarized in Table 2. The most common signs were ascites, pleural calcification/plaques, and omental and peritoneal involvement (100\%, $51.4 \%, 37.2 \%, 31.4 \%$, respectively). During the follow-up, the direct invasion of adjacent abdominal organs and/or distant metastases were diagnosed in 16 patients as follows: liver (nine), liver and spleen (two), lung (one), liver and lung (three) or liver, spleen, lung and bone (one).

Prognostic factors affecting survival. The mean survival time was $16.2 \pm 12.9$ (range 3-52) months. During the followup, 32 of the patients died. Results of survival analyses revealed that age $>60$ years $(p=0.019)$, duration of asbestos exposure $>20$ years $(\mathrm{p}=0.033)$, and ECOG PS of $3(\mathrm{p}=0.000)$ were associated with poor MPM prognosis (Table 3 ).

\section{Discussion}

Prognostic factors are important for the management of MPM as they can help clinicians with both the prognosis of the disease and the treatment plan [4]. In this study, the relationship between survival and demographic data, duration of environmental asbestos exposure, histopathological type, ECOG PS and systemic chemotherapy was determined in patients with MPM. Poor MPM prognosis was found to be associated with the following factors: being older than 60 , having been exposed to environmental asbestos for more than 20 years and having a poor PS (ECOG 3 ).

There are conflicting results regarding the relationship between survival and age in patients with MPM. While some studies have rejected the existence of any relationship between age and survival in MPM patients, others have suggested that patients younger than 60 had a longer survival time $[4,5,7]$. Contrary to these earlier studies, one study reported that younger patients had a poorer survival than their older counterparts. This contradiction has been attributed to the fact that malignant histopathological type (nonepithelioid) mesothelioma occurs more frequently in younger patients [8]. In the present study, patients who were 60 years of age or younger had a longer survival than did patients over the age of 60 . This may be because there were a greater number of patients less than 60 years of age than those over the age of 60 in our study, and younger patients were able to receive systemic chemotherapy since they often had a better PS.
Table 2. Initial radiological findings of the 35 patients with malignant peritoneal mesothelioma

\begin{tabular}{|c|c|c|c|}
\hline Findings & & $\mathrm{n}$ & $\%$ \\
\hline \multirow[t]{3}{*}{ Pleural calcification/plaques } & yes & 18 & 51.4 \\
\hline & no & 4 & 11.4 \\
\hline & unknown & 13 & 37.1 \\
\hline \multirow[t]{2}{*}{ Pleural fluid (US or CT) } & yes & 12 & 34.3 \\
\hline & no & 23 & 65.7 \\
\hline \multirow[t]{3}{*}{ Pleural thickening } & yes & 7 & 20.0 \\
\hline & no & 15 & 42.9 \\
\hline & unknown & 13 & 37.1 \\
\hline \multirow[t]{3}{*}{ Ascites (US) } & slight & 4 & 11.4 \\
\hline & moderate & 18 & 51.5 \\
\hline & extensive & 13 & 37.1 \\
\hline \multirow[t]{2}{*}{ Ascites (CT) } & yes & 24 & 68.6 \\
\hline & unknown & 11 & 31.4 \\
\hline \multirow[t]{3}{*}{ Omental involvement } & yes & 13 & 37.2 \\
\hline & no & 11 & 31.4 \\
\hline & unknown & 11 & 31.4 \\
\hline \multirow[t]{3}{*}{ Peritoneal involvement } & yes & 12 & 34.3 \\
\hline & no & 12 & 34.3 \\
\hline & unknown & 11 & 31.4 \\
\hline \multirow[t]{3}{*}{ Omental caking } & yes & 9 & 25.7 \\
\hline & no & 15 & 42.9 \\
\hline & unknown & 11 & 31.4 \\
\hline \multirow[t]{3}{*}{ Mesentery involvement } & yes & 7 & 20.0 \\
\hline & no & 17 & 48.6 \\
\hline & unknown & 11 & 31.4 \\
\hline \multirow[t]{3}{*}{ Abdominal adenopathy } & yes & 6 & 17.1 \\
\hline & no & 18 & 51.5 \\
\hline & unknown & 11 & 31.4 \\
\hline
\end{tabular}

US: Ultrasound, CT: Computed tomography.

Table 3. Analysis of the prognostic factors influencing the survival of malignant peritoneal mesothelioma patients

\begin{tabular}{lcc}
\hline Variables & $\begin{array}{c}\text { Median survival } \\
\text { (months) }\end{array}$ & $\mathrm{p}^{*}$ \\
\hline Age group (years) & 19.6 & 0.019 \\
$\leq 60$ & 10.3 & \\
$>60$ & 13 & NS \\
Sex difference & 20 & \\
$\quad$ Female & & \\
Male & 24.3 & 0.033 \\
Duration of asbestos exposure (years) & 12.1 & \\
$\leq 20$ & 15.7 & NS \\
$\quad$ 20 & 11 & \\
Histopathological type & & \\
Epithelial & 17.6 & $<0.001$ \\
$\quad$ Others & 19.5 & \\
Performance status & 5.5 & \\
ECOG 1 & & \\
ECOG 2 & 19 & \\
ECOG 3 & 8.4 & \\
Yes & & \\
No & &
\end{tabular}

${ }^{\star}$ Stratified Log Rank test, NS: Not significant. 


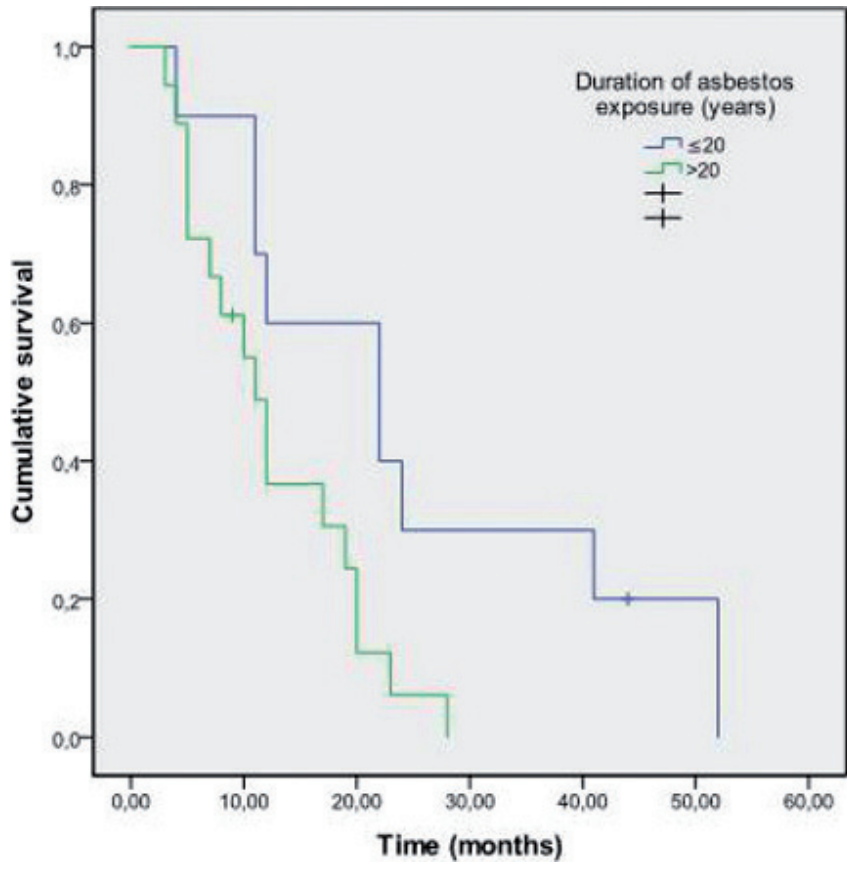

Figure 1. Survival curves according to the duration of environmental asbestos exposure the patients with peritoneal mesothelioma.

The strong relationship between asbestos exposure and the development of mesothelioma at any location is well known, and the latency period is on average 30 years. In industrialized countries, occupational asbestos exposure has been shown to play a significant role in the development of MPM [9]. However, some studies have indicated an increased risk of MPM with non-occupational exposure (i.e. household or residential exposure), especially in developing countries [10,11]. Most researchers agree that asbestos fibers reach the peritoneal cavity in the pathogenesis of MPM by travelling to membrane by means of the lymphatic system following inhalation [12]. High cumulative asbestos exposure is a major risk factor for both pleural and peritoneal mesothelioma [3]. However, asbestos-induced MPM usually requires a higher cumulative dose than its pleural counterpart [13]. It is well known that people have been exposed to environmental asbestos in some rural areas of the Southeast Anatolia Region of Turkey due to its geographical structure [14]. In the present study, the rate of asbestos exposure was much higher (82.9\%) than that reported in the literature, and survival was worse in those with more than 20 years of exposure (Figure 1).

While pleural mesothelioma mainly affects men (male to female ratio: 4-5 to 1), the male/female ratio is much smaller in MPM [1]. In studies of pleural and peritoneal mesothelioma conducted in developed countries, the male gender was found to be associated with a worse survival $[4,15]$. One of the plausible epidemiologic differences between men and women is the probability of asbestos exposure. Direct exposure to asbestos, which was less obvious in women, was a causative factor in men. A study conducted in a country with high rates of asbestos exposure revealed that the rate of asbestos exposure among men was $88 \%$ for pleural mesothelioma and $58 \%$ for peritoneal mesothelioma. These rates were $23 \%$ for both types of mesothelioma in women [9]. In contrast to studies conducted in developed countries, no significant difference was found between sex and survival in the presented study. This may be because both sexes are exposed to asbestos long-term, since it is typically used for domestic purposes such as painting, plastering and ceiling coating [16]. This study suggests that sex is not the sole influential factor in the development and prognosis of MPM, and that the duration of exposure and cumulative dose are more important factors.

Common complaints of patients with MPM include abdominal distension, abdominal pain, nausea, anorexia and weight loss. Abdominal distension is the most common initial symptom, and it is present at diagnosis in $30-80 \%$ of the cases. Abdominal pain is the second most frequent initial symptom, and it occurs in $27-58 \%$ of the cases. Many patients with an advanced disease burden due to the nonspecific nature of the symptoms [17]. In the presented study, the most frequent symptoms were abdominal distension and/or pain (97\%) and weight loss (48\%).

In studies regarding pleural mesothelioma, high platelet and WBC count, high levels of serum LDH and CRP, and low levels of hemoglobin have been associated with poor prognosis $[4,18]$. To our knowledge, there is no study in the literature exploring biochemical prognostic markers in patients with MPM. However, we did not find any significant relationship between survival and the laboratory parameters that were evaluated in our study. This may be because there was a lower number of patients in this study as compared to those regarding pleural mesothelioma.

Computed tomography is the most useful initial diagnostic tool for mesothelioma. Moderate to extensive (or rarely, massive) ascites is present in $60-100 \%$ of the newly diagnosed patients. The majority of patients with MPM present with diffuse peritoneal involvement. Other findings include omental caking/thickening, scalloping, or direct invasion of intraabdominal organs, such as the liver [19]. In the present study, ascites was detected at moderate levels by abdominal ultrasound in all of the patients. The most common finding in patients who had abdominal CT was omental involvement, and the second most common finding was peritoneal involvement. Half of the patients who had thoracic CT showed evidence of pleural calcification/plaques. Direct invasion of adjacent abdominal organs and/or distant metastasis occurred in 16 patients, which was observed during follow-up.

Pleural plaque is the most common radiological manifestation of asbestos exposure. The presence of calcification suggests a benign process. Calcified plaques rarely occur in patients with MPM, and because of this, there are a limited number of published studies investigating thoracic changes 
(pleural changes and effusion) in MPM. Calcified plaques are less frequent in MPM than in pleural mesothelioma, but calcified pleural plaques and other signs associated with asbestos exposure may exist in the chest in up to $50 \%$ of patients with MPM [20]. In a previously published study, pleural changes, such as calcification or thickening, were found in a high proportion (65\%) of patients with MPM [21]. In the present study, radiological evaluation revealed evidence of pleural calcification/plaques in $51 \%$ of the patients and pleural thickening in $20 \%$, which is in accordance with the literature. The presence of pleural plaques may be a guide in the differential diagnosis of MPM (to differentiate from such diseases as peritonitis carcinomatosa and ovarian carcinoma) and may facilitate the establishment of a specific diagnosis.

Eastern Cooperative Oncology Group scoring is a widely used system for the assessment of functional status in mesothelioma patients [7]. Poor prognosis is associated with poor PS, and ECOG PS is one of the most significant predictors of poor prognosis [4]. One study found that patients who scored between 2-4 on the ECOG scale had shorter survival times when compared to those with score between 0-1 [5]. According to the records, our patients with score 1,2 or 3 at the time of diagnosis, and their median survival was 17.6, 19.5 and 5.5 months, respectively. Survival was obviously shorter in those who had an ECOG score of 3.

There is still no consensus on what the optimal MPM treatment should be. For many years, systemic chemotherapy was an important treatment option. Today, platinum-based combination chemotherapy regimens, particularly the combination of cisplatin and pemetrexed, are recommended because of their positive impact on the survival of mesothelioma patients $[22,23]$. In the present study, receiving systemic chemotherapy treatment had no effect on survival. Although patients who received combination chemotherapy seemed to have a relatively longer survival than those who received only supportive care, this result was not significant when age was included as a confounding factor using the stratified Log Rank test. Younger patients may have received chemotherapy due to their better ECOG PS than the older patients.

The limitations in this study arise from its retrospective nature. For example, we could not find imaging results in some files for patients who were referred to our hospital by some other centers following examination.

In conclusion, we determined that age, duration of asbestos exposure and ECOG PS were associated with MPM prognosis. Since the latency period of asbestos exposure is very long, and since asbestos exposure is the most important risk factor for this disease, MPM will continue to appear in the coming years. Therefore, effective measures should be taken to protect those who are still at risk, and criteria should be developed for an early diagnosis and optimal treatment for those who develop the disease. Lastly, prospective studies are needed to determine the factors related to the prognosis of this disease that exhibit a heterogeneous clinical behavior.
Acknowledgements: This submission has not been published anywhere previously and is not simultaneously being considered for any other publication. The authors report no declaration conflict of interests.

\section{References}

[1] TETA MJ, MINK PJ, LAU E, SCEURMAN BK, FOSTER ED. US mesothelioma patterns 1973-2002: indicators of change and insights into background rates. Eur J Cancer Prev 2008; 17: 525-34. http://dx.doi.org/10.1097/CEJ.0b013e3282f0c0a2

[2] BOFFETTA P. Epidemiology of peritoneal mesothelioma: a review. Ann Oncol 2007; 18: 985-90. http://dx.doi.org/ 10.1093/annonc/mdl345

[4] STEELE JP, KLABATSA A, FENNELL DA, PALLÄSKA A, SHEAFF MT et al. Prognostic factors in mesothelioma. Lung Cancer 2005; 49: 49-52. http://dx.doi.org/10.1016/ j.lungcan.2005.03.009

[5] SEBBAG G, YAN H, SHMOOKLER BM, CHANG D, SUGARBAKER PH. Results of treatment of 33 patients with peritoneal mesothelioma. British Journal of Surgery 2000; 87: 1587-93. http://dx.doi.org/10.1046/j.1365-2168.2000.01571.x

[6] OKEN MM, CREECH RH, TORMEY DC, HORTON J, DAVİS TE et al. Toxicity And Response Criteria Of The Eastern Cooperative Oncology Group. Am J Clin Oncol 1982; 5: 649-55. http://dx.doi.org/10.1097/00000421-198212000-00014

[7] MUSK AW, OLSEN N, ALFONSO H, REİD A, MINA $\mathrm{R}$ et al. Predicting survival in malignant mesothelioma. Eur Respir J 2011; 38: 1420-4. http://dx.doi.org/10.1183/ $\underline{09031936.00000811}$

[8] TUDOR EC, CHUA TC, LIAUW W, MORRIS DL. Risk factors and clinicopathological study of prognostic factors in the peritoneal mesothelioma. Am Surg 2010; 76: 400-5.

[9] SPIRTAS R, HEINEMAN EF, BERNSTEIN L, BEEBE GW, KEEHN RJ et al. Malignant mesothelioma: attributable risk of asbestos exposure. Occup Environ Med 1994; 51: 804-11. http://dx.doi.org/10.1136/oem.51.12.804

[11] METINTAS M, METINTAS S, AK G, ERGINEL S, ALATAS F et al. Epidemiology of pleural mesothelioma in a population with non-occupational asbestos exposure. Respirology 2008; 13: 117-21. http://dx.doi.org/10.1111/j.1440-1843.2007.01187.x

[12] MISEROCCHI G, SANCINI G, MANTEGAZZA F, CHIAPPINO G. Translocation pathways for inhaled asbestos fibers. Environ Health 2008; 7: 4. http://dx.doi.org/10.1186/1476$\underline{\text { 069X-7-4 }}$

[13] BERRY G, REID A, ABOAGYE-SARFO P, DE KLERK NH, OLSEN NJ et al. Malignant mesotheliomas in former miners and millers of crocidolite at Wittenoom (Western Australia) after more than 50 years follow-up. Br J Cancer 2012; 106: 1016-20. http://dx.doi.org/10.1038/bjc.2012.23

[14] YAZICIOGLU S. Pleural calcification associated with exposure to chrysotile asbestos in southeast Turkey. Chest 1976; 70: 43-7. http://dx.doi.org/10.1378/chest.70.1.43

[15] YAN TD, POPA E, BRUN EA, CERRUTO CA, SUGARBAKER PH. Sex difference in diffuse malignant peritoneal mesothelioma. Br J Surg 2006; 93: 1536-42. http://dx.doi. org/10.1002/bjs. 5377 
[16] SENYIGIT A, BABAYIGIT C, GOKIRMAK M, TOPCU F, ASAN E et al. Incidence of malignant pleural mesothelioma due to environmental asbestos fiber exposure in the southeast of Turkey. Respiration 2000; 67: 610-4. http://dx.doi. org/10.1159/000056289

[17] SUGARBAKER PH, WELCH LS, MOHAMED F, GLEHEN O. A review of peritoneal mesothelioma at the Washington Cancer Institute. Surg Oncol Clin N Am 2003; 12: 605. http:// dx.doi.org/10.1016/S1055-3207(03)00045-0

[18] TANRIKULU AC, ABAKAY A, KAPLAN MA, KUCUKONER M, PALANCI Y et al. A Clinical, Radiographic and Laboratory Evaluation of Prognostic Factors in 363 Patients with Malignant Pleural Mesothelioma. Respiration 2010; 80: 480-7. http://dx.doi.org/10.1159/000321370

[19] PARK JY, KIM KW, KWON HJ, PARK MS, KWON GY et al. Peritoneal mesotheliomas: clinicopathologic features, CT findings, and differential diagnosis. AJR Am J Roentgenol 2008; 191: 814. http://dx.doi.org/10.2214/AJR.07.3628
[20] GUEST PJ, REZNEK RH, SELLESLAG D, GERAGHTY R, SLEVIN M. Peritoneal mesothelioma: the role of computed tomography in diagnosis and follow up. Clin Radiol 1992; 45: 79-84. http://dx.doi.org/10.1016/S0009-9260(05)80059-5

[21] KEBAPCI M, VARDARELI E, ADAPINAR B, ACIKALIN M. CT findings and serum ca 125 levels in malignant peritoneal mesothelioma: report of 11 new cases and review of the literature. Eur Radiol 2003; 13: 2620-6. http://dx.doi. org/10.1007/s00330-003-1851-6

[22] BERK S, DOGAN OT, KİLİCKAP S, EPOZTURK K, AKKURT I et al. Clinical characteristics, treatment and survival outcomes in malignant mesothelioma: eighteen years ${ }^{\varsigma}$ experience in Turkey. Asian Pac J Cancer Prev 2012; 13: 5735-9. http://dx.doi.org/10.7314/APJCP.2012.13.11.5735

[23] MIRARABSHAHII P, PILLAI K, CHUA TC, POURGHOLAMI MH, MORRIS DL. Diffuse malignant peritoneal mesothelioma - An update on treatment. Cancer Treat Rev 2012; 38: 605-12. http://dx.doi.org/10.1016/j.ctrv.2011.10.006 